\title{
Features of Our SEM Transmission Diffraction Sub-stage with 6-axis Sample Control and a Camera with Variable Camera Length
}

\author{
Johannes Müller, Benedikt Haas, Wouter Van den Broek, Sherjeel Shabih and Christoph T. Koch \\ Humboldt-Universität zu Berlin, Berlin, Berlin, Germany
}

With the development of fast electron sensitive cameras, a wide range of diffraction-based techniques has recently been developed for 4D-STEM [1], many of these techniques can also be employed in conventional SEMs and FIB/SEMs by adding a fast camera inside the sample chamber [2]. We built a diffraction sub-stage for SEMs and FIB/SEMs which can acquire fast and with the beam scan synchronized diffraction patterns in transmission. This sub-stage can also tilt and shift the sample piezodriven in 6 dimensions and adjust the camera length by moving the camera relative to the sample with a linear stage. This system is simpler and more flexible compared to dedicated transmission electron microscopes, has a larger sample chamber and less operating costs. Additionally, the low acceleration voltages $(0.5 \mathrm{kV}$ to $30 \mathrm{kV})$ of SEMs and FIB/SEMs result in stronger scattering as well as reduced knockon damage compared to higher voltages. With their good electron optics, SEMs and FIB/SEMs are an excellent platform for investigating beam sensitive materials like hybrid inorganic/organic systems, 2D materials, FIB lamellas and other thin samples with diffraction based methods. We present our plug and play sub-stage that can easily be transferred between SEMs and FIB/SEMs in more detail and show a summary of our results.

Our goal is to have a simple system for applying and developing dose-efficient low voltage electron diffractions techniques, such as advanced variants of 4D-STEM to answer questions related to thin bulk and low dimensional samples. With the existing detectors like EDX, the addition of fast electron diffraction and the 6-axis sample control, we have several tools at our hand to map material parameters from $\mathrm{nm}$ to $\mathrm{cm}$ scale. This tool is a handy addition to labs that synthesize thin samples and have access to a SEM or FIB/SEM but don't want to invest in dedicated TEMs giving them the possibility to investigate their materials in-house.

The custom setup consists of a Zeiss GeminiSEM 500 with a compact $2.5 \times 2.5 \times 1.1 \mathrm{~cm}^{3}$ scintillator-based camera, a hexapod sub-stage, a linear positioner to move the camera, sample holders, a cooling unit, a scan generator plus a measurement $\mathrm{PC}$. The hexapod shift ranges $(\mathrm{x}, \mathrm{y}, \mathrm{z})$ are up to $1.6 \mathrm{~cm}$ covering an area of $1.6 \times 1.6 \mathrm{~cm}^{2}$, and the tilt ranges (a,b) are up to $24^{\circ}$ with the standard holder. The normal sample holders can fit TEM grids and are mounted to the movable platform of the hexapod. The camera is fiber-coupled and the camera length ranges from 5 to $40 \mathrm{~mm}$ by moving the camera with a software controlled linear stage, there are no post-specimen lenses. Our stage has no influence on the chamber vacuum due to the used low outgassing materials. We have full scripting access to our hardware via C++/C\# DLLs and developed a scan generator to synchronize the beam position, the camera exposure and the fast beam blanker to get 160fps at 1200x1200 pixels and up to 2000fps at 64x64 pixels. We can scan arbitrary patterns with our scan generator like interlaced scanning to reduce beam damage between neighbouring scan position. The SEM allows to control the convergence angle and the current up to 20nA allowing for the acquisition of spot patterns, CBED patterns, Ronchigrams and shadow images. We use the open-source Python-based platform Nion Swift as GUI.

We applied our diffraction setup to map material properties of bulk, $2 \mathrm{~d}$ and $1 \mathrm{~d}$ samples like layer thickness, crystal orientations, defects, strain, electric fields, magnetic fields, unit cell parameters, crystallinity, material composition and other crystallographic information as well as electric fields of biased electrodes. 
With the addition of fast transmission electron diffraction and 6-axis sample control to SEMs and FIB/SEMs, we have a low cost and simply working tool at our hand for investigating thin bulk and low dimensional materials with many exciting diffraction based methods.
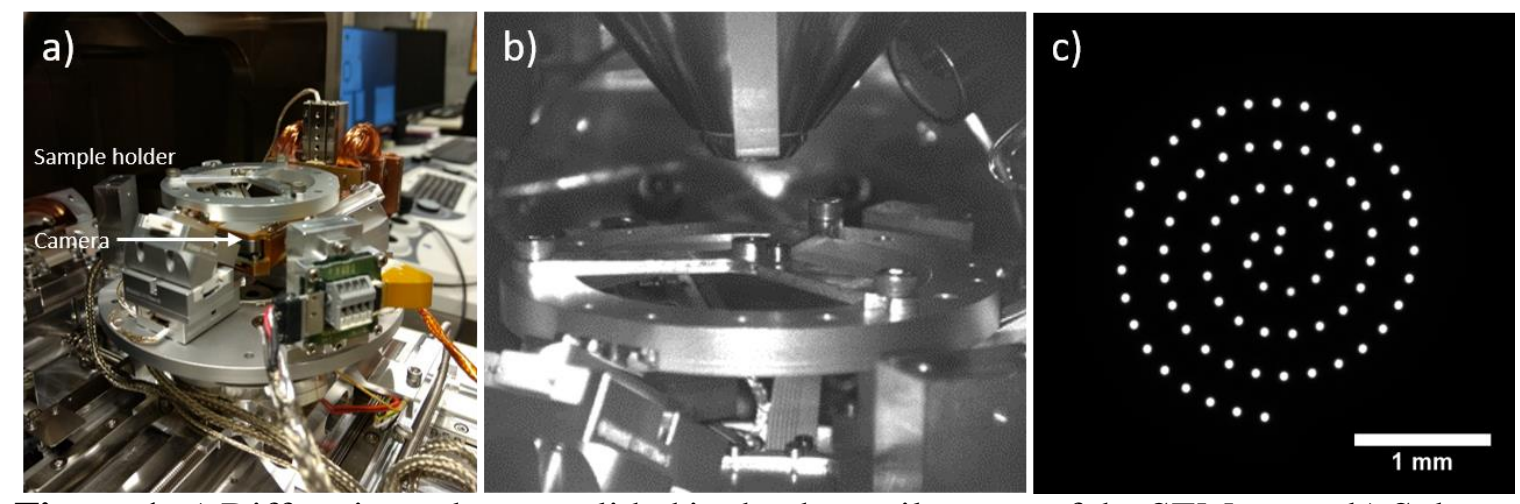

Figure 1. a) Diffraction sub-stage slided in the dovetail mount of the SEM stage. b) Sub-stage in our SEM. c) Integrated spiral scan pattern scanned without a sample across the camera.

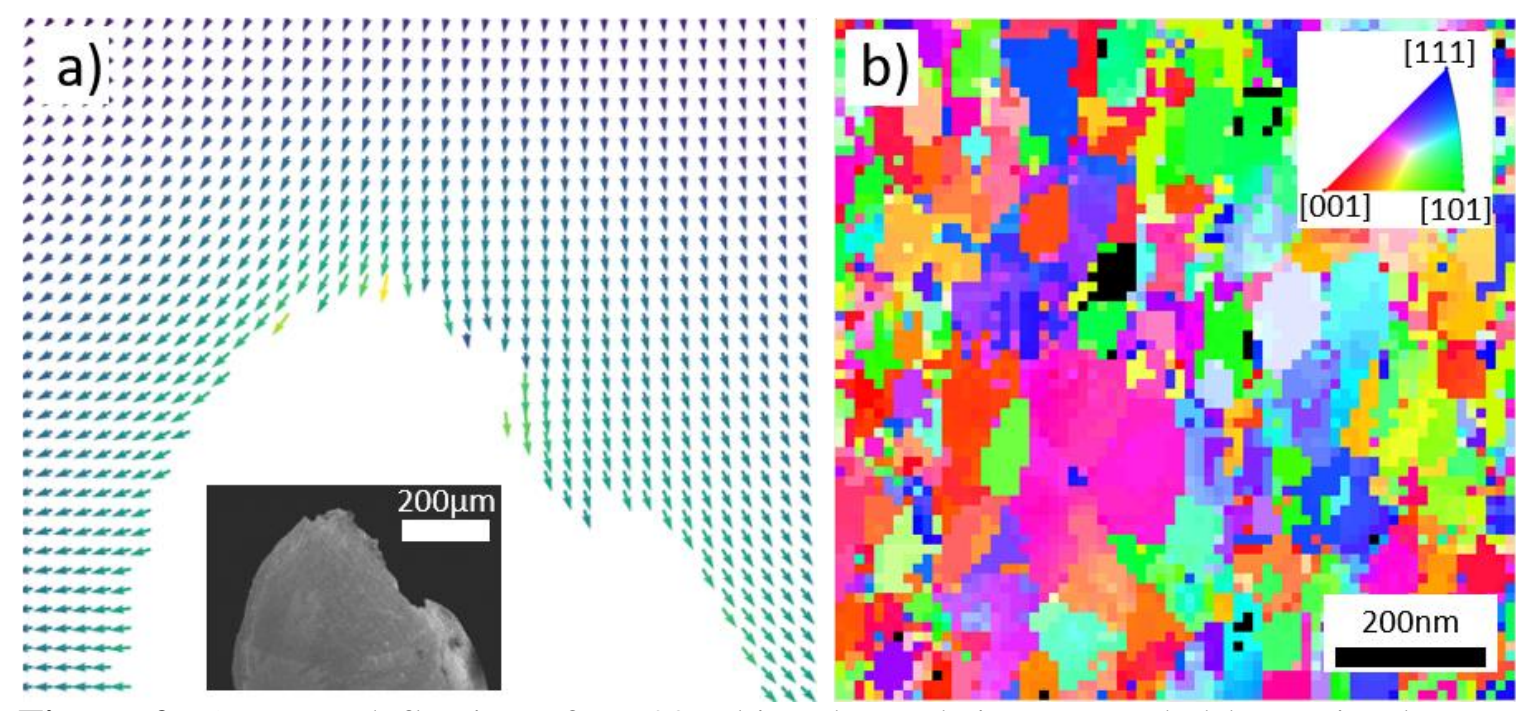

Figure 2. a) Beam deflection of a $+80 \mathrm{~V}$ biased metal tip surrounded by a circular grounded aperture measured with center of mass, the maximum deflection is $13.1 \mathrm{mrad}$ at $6.5 \mathrm{keV}$ electron energy. The inset shows a SE image of the tip. b) Grain orientation map of an aluminum sample.

\section{References}

[1] C. Ophus, Microsc. Microanal. 25 (2019) 563

[2] B. Haas, Imaging \& Microscopy 21 (2019) 35

We acknowledge financial support by the German Research Foundation (DFG grant nrs. KO 2911/12-1 \& KO 2911/13-1, as well as Projektnummer 182087777 - SFB951). We thank Harald Niebel, Björn Gamm, Markus Boese from Carl Zeiss Microscopy GmbH for help with controlling the SEM. 